\title{
Yesterday, Today and Tomorrow of Mycology
}

\section{Habip Gedik*}

Department of Infectious Diseases and Clinical Microbiology, Ministry of Health Bakırkoy Training and Research Hospital, Istanbul, Turkey

${ }^{*}$ Corresponding author: Habip Gedik, Department of Infectious Diseases and Clinical Microbiology, Ministry of Health Bakırkoy Training and Research Hospital, Istanbul, Turkey, Tel: 009050533627 70; E-mail: habipgedik@yahoo.com

Received date: August 04, 2016; Accepted date: August 16, 2016; Published date: August 26, 2016

Citation: Habip G (2016) Yesterday, Today and Tomorrow of Mycology. Med Mycol Open Access 2: 3. doi: 10.21767/2471-8521.1000e1.

Copyright: (C) 2016 Habip G. This is an open-access article distributed under the terms of the Creative Commons Attribution License, which permits unrestricted use, distribution, and reproduction in any medium, provided the original author and source are credited.

\section{Editorial}

Fungi are a part of environmental life. The use of yeasts dates back to Sumeria 7000 B.C for beer. Fungi essentially produce beer, wine, citric acid, single cell protein, fodder yeast, and baker's yeast. Fungi had become objects of interest with a growing awareness as a material for botanical investigation at the beginning of 1900s. It had contributed to development of Biology, and Botany in the first fifty years of the 20th century. Fungi are suitable material for investigation, as they can be cultured under controlled conditions within a short time and remain stable for long-term investigations as well. Diversity of physiological activity, eighty to ninety thousand species within their versatility according to taxonomic prejudice, from singlecelled forms and simple aggregates to branching filamentous pattern, sexual and asexual reproduction, and fusion between filaments had made them study subject for scientists. Progress in mycological work contributed to Botany and increased the interest in this area [1]. However, fundamental of mycology was laid before. Dr. John Gill was firstly reported Mycetoma as Madura foot by in a dispensary report of the Madras Medical Service of British Army in India in 1842. Nevertheless, French missionaries defined a disease similar to mycetoma in Pondicherry in 1714. Godfrey, a surgeon, described mycetoma in 1846 as Morbus tuberculosis paddies. Carter published the monograph in 1874 as "on mycetoma or the fungus disease of India" [1]. Rhinosporidiosis, Tinea capitis, Chromoblastomycosis, pulmonary mycoses, and animal to man dermatomycoses were presented as published studies in India as well [2-6].

Fungal infections had emerged as superficial and cutaneous mycoses before. Pityriasis versicolor, Seborrhoeic dermatitis including Dandruff and Follicular pityriasis (Malassezia spp.), Tinea nigra (Exophiala werneckii), White piedra (Trichosporon spp.), and black piedra (Piedraia hortae) are encountered as superficial mycoses. Dermatophytosis in the scalp, glabrous skin and nails (Nannizzia, Trichophyton, Arthroderma, Lophophyton, Microsporum, Epidermophyton), Candidiasis in the skin, mucous membranes and nails (Candida, Debaryomyces, Meyerozyma, Pichia, Kluyveromyces, etc.), and Dermatomycosis (Non-dermatophyte molds, Neoscytalidium, Scopulariopsis) are cutaneous mycoses. Subcutaneous and dimorphic systemic mycoses had taken places in mycology after regional cases were reported and geographically emerged.

Candidiasis (Candida, Debaryomyces, Kluyveromyces, Meyerozyma, Pichia, etc.), Cryptococcosis (Cryptococcus neoformans), Aspergillosis (Aspergillus fumigatus, Aspergillus niger, etc.), Pseudallescheriasis (Scedosporium, Lomentospora), Zygomycosis (Mucormycosis, Rhizopus, Mucor, Rhizomucor, Lichtheimia, etc.), Hyalohyphomycosis (Penicillium, Paecilomyces, Beauveria, Fusarium, Scopulariopsis, etc.), and Phaeohyphomycosis (Cladophialophora, Exophiala, Bipolaris, Exserohilum, etc.) have been emerging in patients who have HIV, or receive post-transplantation chemotherapy, aggressive cancer and the use of broad-spectrum antibiotics, immunosuppressives, corticosteroids or undergo invasive procedures that impair the physiological barriers.

Candida species is a member of the normal flora of gastrointestinal tract and skin. Candidemia commonly develops in immuncompromised patients in relation with colonization in the body sites, broad-spectrum antibiotic use, and impairment of physiological barriers in the gastrointestinal system [7,8]. Candidemia was reported as fourth most common cause of nosocomial bloodstream infections in the United States and from fourth to seventh cause in Europe $[9,10]$. The incidence rates of fungemia were reported to be from 0.28 to 0.96 per 1,000 admissions in the United States and from 0.2 to 0.38 in Europe, as the rates varied from 1.2 to 5.3 (0.2-0.5 cases per 1,000 patient-days) in Latin America $[9,11]$. Urinary catheter, immunosuppressive therapy, hypoalbuminemia and APACHE II score ( $\geq 16$ ) are mortalityrelated risk factors. Follow-up in the intensive care unit (ICU), central venous catheterization, mechanical respiratory support, urinary catheterization, malignancy, acute or chronic renal failure, total parenteral nutrition (TPN), any surgical procedures, non-response to antifungal treatment, comorbidity, high Charlson index, shock were presented as mortality related risk factors in the candidemia cases [12-15]. Extensive azole use, fungal ecosystem, geographic location and conditions of hospitals are associated with diversity of species which causes candidemia. C. parapsilosis is related to vascular catheters and parenteral nutrition [16]. C. tropicalis is related to cancer and neutropenia [17]. C. krusei and C. glabrata are commonly associated with a history of exposure to azoleantifungal drugs [17]. Although removal of catheter after 
identification of candidemia was recommended in the previous studies, Nucci and colleagues reported that catheter removal have no effect on overall treatment response, mortality and mycological eradication rates by 24 or 48 hours after treatment initiation $[11,18,19]$. Catheter should be removed taking into account benefits and harms in the patient. Prognosis is related to severity of illness and underlying conditions. Caspofungin, amphotericin B, isovuconazole, anidulafungin, and voriconazole should be initiated if patien is hemodynamically unstable, or has one of those factors including central venous catheter, a history of exposure to azole-antifungal drug. Antifungal treatment should be administered at least two weeks after last positive blood culture [13]. Crude-30 day mortality rates were revealed to be from $20 \%$ to $63 \%$ in the candidemia cases $[12,13]$. Poor prognosis is associated with delayed or inappropriate antifungal treatment [20]. Attributed mortality rates were reported to be from $15 \%$ to $25 \%$ in the adults [21]. Antifungal treatment should be administered as soon as possible after diagnosis of fungemia.

Aspergillosis is defined as a spectrum of diseases caused by members of the genus Aspergillus in the humans and animals. These include mycotoxicosis owing to ingestion of contaminated foods; allergy to the presence of conidia or transient growth of the organism; colonisation without extension in preformed cavities and debilitated tissues; invasive, inflammatory, granulomatous, necrotising disease of lungs, and other organs; and systemic and fatal disseminated disease. The type of disease and severity depends on the physiological state of the host. The etiological agents are Aspergillus fumigatus complex, A. flavus complex, $A$. niger complex, $A$. nidulans and $A$. terreus complex [22]. Definitive diagnosis of invasive aspergillosis (IA) is performed with the histopathologic demonstration of tissue invasion by hyphae. However, biopsy is commonly not practical owing to the risks of complications. A thorax computed tomographic (CT) scan is recommended if there is a clinical thought for invasive pulmonary aspergillosis (IPA). Routine use of contrast for a suspicion of IPA is not recommended. Thorax CT scan is recommended after a minimum of two weeks of treatment to evaluate the response of treatment. Earlier appraisal is indicated if the patient clinically worsens. Obtaining sputum and/or bronchoalveolar lavage (BAL) specimens for fungal staining and culture and serum biomarkers (galactomannan (GM) and beta-D-glucan assays) are used for the diagnosis of IA. Galactomannan antigen testing should be performed in BAL samples. Diagnosis of the invasive fungal infections (IFIs) is still a big drawback in spite of Aspergillus galactomannan antigen test with a sensitivity rate of $58 \%-65 \%$ and a specificity rate of $65 \%-95 \%$ (for patients who have hematologic malignancy or undergo allogeneic hematopoietic stem cell transplant (HSCT) and CT scanning [23]. Mortality has reduced $5 \%$ in patients with solid tumors, and $11 \%$ in cases with hematological malignancies after new tests and modalities [24]. A positive microbiological culture and fungal stain of sputum should be stimulating for IA in patients at risk. The serum GM test is relatively specific for IA, and adequate evidence of IPA. BAL and serum GM test is useful as an accurate marker for the diagnosis of IA. GM is not recommended for routine blood assessment in patients receiving antifungal therapy or prophylaxis for mold-fungi. In contrast, positive serum beta-Dglucan test can be found in various invasive fungal infections. However, clinical utility of blood-based polymerase chain reaction (PCR) is still debated the in diagnosing IA. PCR should be used carefully taking into account clinical context and other diagnostic tests. Antifungal susceptibility testing is not routinely recommended for Aspergillus spp. isolates, unless there is a suspected azole-resistant isolate, or a case who is unresponsive to antifungal agents, or for epidemiological purposes [25]. The overall crude mortality rate (CMR) was $58 \%$, and the CMR was highest for bone marrow transplant recipients $(86.7 \%)$ and for patients with central nervous system or disseminated aspergillosis (88.1\%) [26]. Posaconazole, voriconazole, isovuconazole, and/or micafungin could be used during prolonged neutropenia of patients at high risk for IA [25].

Three major subjects will be issued in mycology: Very highly sensitive and specific tests for accurate and timely diagnosis of IFIs, antifungal resistance, and emerging fungal pathogens. IFIs were confirmed in $4 \%$ of the cases that covered only from $22 \%$ to $34 \%$ of the neutropenic patients who received an antifungal drug or had posessed according to established criteria [27]. New diagnostic tests which provide earlier diagnosis will proceed timely antifungal therapy that increase survival rates. It should be noted that invasive fungal infections can be prevented by healthy anatomical and physiological barriers and immune response. Antifungal drugs alone cannot prevent and treat the invasive fungal infections. Recovery of patients' healthy anatomical and physiological barriers and immune response is essential for cure. Every antimicrobial drug encounters antimicrobial resistance that is developed by concerning microorganisms and this is the need to maintain the life. Development of resistance to existing antifungaldrugs is certainly and inevitable. We need new antifungal drugs for treatment of antifungal-resistant IFIs and emerging pathogens that are resistant to existing antifungal drugs. It is obvious that mycology is open new researches, innovations, projects and studies.

\section{References}

Weston WH (1957) Mycology During the Past Fifty Years. American Journal of Botany 44: 82-87.

2. Allen $D$ (1936) The treatment of rhinosporidiosis in man based on the study of 60 cases. Indian Med Gaz 71: 376-394.

3. Dey NC (1953) A review of ringworm of hair in India. Indian Med. Gaz 88: 194-196.

4. Andleigh (1953) Chromoblastomycosis-review with a favourable case. Indian J Med Sci 7: 409-414.

5. Andleigh (1958) Investigation in the role of fungi in pulmonary diseases in India. Am Rev Tuberculosis 78: 644-646.

6. Chakraborty AN, Ghosh S, Banerjee AK (1953) Review on the study of skin disease in animal communicable to man. Indian Med Gaz 88: 152-153. 
7. Vincent $\mathrm{JL}$, Anaissie $\mathrm{E}$, Bruining $\mathrm{H}$, Demajo $\mathrm{W}$, el-Ebiary $\mathrm{M}$, et al. (1998) Epidemiology, diagnosis and treatment of systemic Candida infection in surgical patients under intensive care. Intensive Care Med 24: 206-216.

8. Kullberg BJ, Sobel JD, Ruhnke M, Pappas PG, Viscoli C, et al. (2005) Voriconazole versus a regimen of amphotericin $B$ followed by fluconazole for candidaemia in nonneutropenic patients: a randomised non-inferiority trial. Lancet 366: 1435-1442.

9. Pappas PG, Kauffman CA (2009) Clinical Practice Guidelines for the Management of Candidiasis: 2009 Update by the Infectious Diseases Society of America. Clin Infect Dis 48: 503-535.

10. Sifuentes-Osornio J, Corzo-Leon DE, Ponce-de-Leon LA (2012) Epidemiology of Invasive Fungal Infections in Latin America. Curr Fungal Infect Rep 6: 23-34.

11. Nucci M, Anaissie E, Betts RF, Dupont BF, Wu C, et al. (2010) Early Removal of Central Venous Catheter in Patients with Candidemia Does Not Improve Outcome: Analysis of 842 Patients from 2 Randomized Clinical Trials. Clin Infect Dis 51 295-303.

12. Erdem I, Oguzoglu N, Ozturk DE, Ozgultekin A, Sengoz IA, et al. (2010) Incidence, etiology and risk factors associated with mortality of nosocomial candidemia in a tertiary care hospital in Istanbul, Turkey. Med Princ Pract 19: 463-467.

13. Jutiamornlerd N, Chusri S, Siripaitoon P (2011) Epidemiology of Candidemia in Songklanagarind Hospital. J Med Assoc Thai 94: 927-232.

14. Diaz-Granados CA, Martínez A, Deaza C, Valderrama S (2008) An outbreak of Candida spp. bloodstream infection in a tertiary care center in Bogotá, Colombia. Braz J Infect Dis 12: 390-394.

15. Yildirmak MT, Gedik H, Simsek F, Iris NE (2013) A: Is Timely and Appropriate Antifungal Drug Enough for Survival of Adult Cases with Candidaemia? Five-year Experience. West Indian Med J 62: 724-730.

16. Clark TA, Slavinski SA, Morgan J, Lott T, Arthington-Skaggs BA, et al. (2004) Epidemiologic and molecular characterization of an outbreak of Candida parapsilosis bloodstream infections in a community hospital. J Clin Microbiol 42: 4468-4472.

17. Bassetti M, Ansaldi F, Nicolini L, Malfatto E, Molinari MP, et al. (2009) Incidence of candidaemia and relationship with fluconazole use in an intensive care unit. J Antimicrob Chemother 64: 625-629.

18. Morgan J, Meltzer MI, Plikaytis BD, Sofair AN, Huie-White S, et al. (2005) Excess mortality, hospital stay, and cost due to candidemia: a case- control study using data from populationbased candidemia surveillance. Infect Control Hosp Epidemiol 26: 540-547.

19. Mermel LA, Allon M, Bouza E, Craven DE, Flynn P, et al. (2009) Clinical Practice Guidelines for the Diagnosis and Management of Intravascular Catheter-Related Infection: 2009 Update by the Infectious Diseases Society of America. Clin Infect Dis 49: 1-45.

20. Kumar A, Ellis P, Arabi Y, Roberts D, Light B, et al. (2009) Initiation of inappropriate antimicrobial therapy results in a fivefold reduction of survival in human septic shock. Chest 136: 1237-1248.

21. Horn DL, Neofytos D, Anaissie EJ, Fishman JA, Steinbach WJ, et al. (2009) Epidemiology and outcomes of candidemia in 2019 patients: data from the prospective antifungal therapy alliance registry. Clin Infect Dis 48: 1695-1703.

22. Richardson MD, Warnock DW (1993) Fungal Infection: Diagnosis and Management. Blackwell Scientific Publications, London.

23. Freifeld AG, Bow EJ, Sepkowitz KA, Boeckh MJ, Ito Jl, et al. (2011) Clinical practice guideline for the use of antimicrobial agents in neutropenic patients with cancer: 2010 update by the Infectious Diseases Society of America. Clin Infect Dis 52: 56-93.

24. Naurois J, Novitzky-Basso I, Gill MJ, Marti Marti FM, Cullen MH, et al. (2010) Management of febrile neutropenia: ESMO Clinical Practice Guidelines. Ann Oncol 21: 252-256.

25. Patterson TF, Thompson GR, Denning DW, Fishman JA, Hadley S, et al. (2016) Practice Guidelines for the Diagnosis and Management of Aspergillosis: 2016 Update by the Infectious Diseases Society of America. Clin Infect Dis 63: 1-60.

26. Lin SJ, Schranz J, Teutsch SM (2001) Aspergillosis case-fatality rate: systematic review of the literature. Clin Infect Dis 32: 358-366.

27. Walsh TJ, Finberg RW, Arndt C, Hiemenz J, Schwartz C, et al. (1999) Liposomal amphotericin B for empirical therapy in patients with persistent fever and neutropenia. National Institute of Allergy and Infectious Diseases Mycoses Study Group. N Engl J Med 340: 764-771. 